\title{
EXPONENTIAL SOLUTION OF A DIFFERENTIAL EQUATION BETWEEN OPERATORS
}

\author{
H. LUNA-GARCÍA AND LUZ MARÍA GARCÍA-CRUZ
}

(Communicated by Xiao-Jun YANG)

\begin{abstract}
ABSTRACT: In this paper we obtain an authentically exponential solution to the differential equation $\frac{d Y(t)}{d t}=A(t) Y(t)$, where $Y(t)$ and $A(t)$ are linear operators with the initial condition $Y(0)=I$, and $I$ is the identity operator. Then, the solution was applied to calculate the vector triad: tangent, normal and binormal in terms of the arc length of a curve.
\end{abstract}

\section{INTRODUCTION}

Studying a homogeneous system of differential equations, leads immediately to the differential equation $\frac{d Y(t)}{d t}=A(t) Y(t)$. Examples of this relation are described in the evolution equation and the Bloch equation in quantum mechanics. Also, it can be found by studying the rotations in classical mechanics, as well as, other topics of the mathematical physics. The evolution equation has previously solved by J. Schwinger [1], F. J. Dyson [2], R. P. Feynman [3] among others. The solutions found are not expressed as a true exponential form. However, H. Luna and O. Chavoya [4], found a truly exponential solution in a particular case when $A(t)$ is independent of the parameter $t$.

Also, W. Magnus [5] solved the same equation in an approximate exponential giving a solution where the exponent was expressed in terms of multiple commutators.

Its solution makes use of super multiple commutators operators $\{$,$\} and the$ derivative of polarization $\left(y \frac{\partial}{\partial x}\right)$.

This paper is divided into five parts: This introduction, section 2 illustrates the solution given by W. Magnus, in section 3, our solution is obtained truly exponential, and it is compared with that obtained by Magnus, being the nearest to our solution; section 4 presents the application of our solution to a special case: the

Date: Received: November 15, 2014; Accepted: December 20, 2014.

2010 Mathematics Subject Classification. 00A69, 34A05, $53 \mathrm{Z} 05$.

Key words and phrases. Exponential of a matrix, Lie groups,Frenet-Serret equations.

This article is the written version of author's plenary talk delivered on August 25-28, 2014 at 3rd International Eurasian Conference on Mathematical Sciences and Applications IECMSA-2014 at Vienna, Austria. 
calculation of the triad of vectors tangent, normal and binormal in terms of the arc length. Finally, section 5 presents conclusions and future projects.

\section{SOLUTION OF W. MAGNUS}

His method is as follows. Given the differential equation.

$$
\frac{d Y(t)}{d t}=A(t) Y(t),
$$

it seeks an operator $Y(t)$ in terms of the linear operator $\Omega(t)$ so as to replace in Eq. (2.1) the following is obtained:

$$
\frac{d e^{\Omega(t)}}{d t}=A(t) e^{\Omega(t)} .
$$

It is known that the derivative with respect to $t$ of $e^{\Omega(t)}$ can be expressed in terms of the polarization derivative of the exponential as follows:

$$
\frac{d e^{\Omega(t)}}{d t}=\dot{\Omega}\left(\frac{\partial}{\partial \Omega}\right) e^{\Omega(t)}
$$

Combining Eq. (2.2) and Eq. (2.3), we have

$$
\left(\dot{\Omega} \frac{\partial}{\partial \Omega}\right) e^{\Omega}=A e^{\Omega}
$$

The polarization derivative is then expressed in terms of the super multiple commutators operators $\{$,

$$
\left(\dot{\Omega} \frac{\partial}{\partial \Omega}\right) e^{\Omega}=\left\{\frac{e^{\Omega}-I}{\Omega}, \dot{\Omega}\right\} e^{\Omega},
$$

where $\dot{\Omega}=\frac{d \Omega}{d t}$. From Eq. (2.4) and Eq. (2.5) we obtain

$$
\left\{\frac{e^{\Omega}-I}{\Omega}, \dot{\Omega}\right\}=A,
$$

and multiplying by the inverse follows

$$
\left\{\frac{\Omega}{e^{\Omega}-I},\left\{\frac{e^{\Omega}-I}{\Omega}, \dot{\Omega}\right\}\right\}=\left\{\frac{\Omega}{e^{\Omega}-I}, A\right\},
$$

where

$$
\dot{\Omega}=\left\{\frac{\Omega}{e^{\Omega}-I}, A\right\} .
$$

By using in Eq. (2.8), and the well-known formula [6]

$$
\frac{t}{e^{t}-1}=\sum_{n=0}^{\infty} \frac{B_{n}}{n !} t^{n},
$$

where $B_{n}$ are the Bernoulli numbers, it is possible to write

$$
\frac{d \Omega}{d t}=\sum_{n=0}^{\infty} \frac{B_{n}}{n !}\left\{\Omega^{n}, A\right\},
$$


then integrating Eq. (2.10) from zero to $t$, finally we obtain

$$
\Omega(t)=\sum_{n=0}^{\infty} \frac{B_{n}}{n !} \int_{0}^{t} d \tau\left\{\Omega^{n}(t), A(\tau)\right\}
$$

It is evident from Eq. (2.11) that $\Omega(t)$ is expressed in terms of powers of $\Omega$. Iterating Eq. (2.11) can be found $\Omega(t)$ in terms of $A(t)$ as follows:

$$
\Omega(t)=\int_{0}^{t} d t_{1} A_{1}+\frac{1}{2} \int_{0}^{t} d t_{2} \int_{0}^{t_{2}} d t_{1}\left[A_{2}, A_{1}\right]+\frac{1}{4} \int_{0}^{t} d t_{3} \int_{0}^{t_{3}} d t_{2} \int_{0}^{t_{2}} d t_{1}\left[A_{3},\left[A_{2}, A_{1}\right]\right]+\ldots
$$

where $\left[A_{j}, A_{k}\right]=A_{j} A_{k}-A_{k} A_{j}$ and $A_{j}=A\left(t_{j}\right)$.

While this method has the disadvantage of expressing $\Omega(t)$ always based on $\Omega(t)$ and this difficulty cannot be removed, also has favorable property of displaying the operator $\Omega(t)$ as a function of the multiple commutators of $A(t)$.

Also, R. M. Wilcox [7] solved the differential equation at hand finding the first terms of the previous series. Likewise, Mielnik and Plebañsky [8] solved the evolution equation with an algorithm similar to that used for E. B. Dynkin [9] in the search for a solution to the famous Campbell-Baker-Hausdorff's problem. Unfortunately, the method of Mielnik and Plebañsky is very complicated, for this, as mentioned in the summary, aims to provide a solution truly exponential given differential equation.

\section{OUR SOLUTION}

Find the exponential solution of the differential equation

$$
\frac{d Y(t)}{d t}=A(t) Y(t)
$$

means finding an operator $\Omega(t)$ in terms of $A(t)$ such that $Y(t)=e^{\Omega}$. Substituting $Y(t)$ in Eq. (3.1) we have

$$
\frac{d e^{\Omega(t)}}{d t}=A(t) e^{\Omega(t)}
$$

but, on the other hand, it is known that

$$
\frac{d e^{\Omega(t)}}{d t}=\int_{0}^{1} d \lambda e^{\lambda \Omega} \dot{\Omega} e^{-\lambda \Omega} e^{\Omega}
$$

where $\lambda$ is a $t$ independent variable and $|\lambda| \leq 1$. Comparing Eq. (3.2) and Eq. (3.3) one has

$$
\int_{0}^{1} d \lambda e^{\lambda \Omega} \dot{\Omega} e^{-\lambda \Omega}=A
$$

It is easy to show that

$$
\dot{\Omega}=e^{-\Omega}\left(\frac{d}{d t}-A\right) \Omega e^{\Omega}
$$


For this purpose we replace Eq. (3.5) in Eq. (3.4) and multiplying on the left by $e^{\Omega(t)}$, lets the following results

$$
\int_{0}^{1} d \lambda e^{\lambda \Omega}\left(\frac{d}{d t}-A\right) \Omega e^{-\lambda \Omega} e^{\Omega}=e^{\Omega} A .
$$

Using the anticommutative property between $e^{\Omega}$ and $A$ and introducing the unit operator $I$, the Eq. (3.6) becomes

$$
\int_{0}^{1} d \lambda e^{\lambda \Omega}\left(\frac{d}{d t}-A\right) \Omega e^{-\lambda \Omega} e^{\Omega}=\int_{0}^{1} d \lambda e^{\lambda \Omega} A e^{-\lambda \Omega}
$$

From the definition of the polarization derivative, the Eq. (3.7) becomes

$$
\left(\frac{d}{d t}-A\right) \Omega \frac{\partial}{\partial \Omega} e^{\Omega}=\left(A \frac{\partial}{\partial \Omega}\right) e^{\Omega}
$$

Identifying coefficients in Eq: (3.8), we obtain

$$
\left(\frac{d}{d t}-A\right) \Omega=A
$$

Therefore

$$
\Omega(t)=\left(\frac{d}{d t}-A\right)^{-1} A
$$

Then, replacing $x=\frac{d}{d t}-A$, and using the polarization derivative, the Eq. (3.10) can be expressed as

$$
x^{-1} A=-A \frac{\partial}{\partial x},
$$

of which,

$$
\left[x, x^{-1} A\right]=A \text {. }
$$

Then, substituting $x$ and $x^{-1}$ in Eq. (3.12) we obtain

$$
\left[\frac{d}{d t}-A, \Omega\right]=A
$$

where this equation represents a differential equation of $\Omega(t)$. From the basic identity

$$
\Omega=e^{\Omega} \Omega e^{-\Omega},
$$

and, $\frac{d e^{\Omega}}{d t}=A e^{\Omega}, \frac{d e^{-\Omega}}{d t}=-e^{-\Omega} A$, one has

$$
\dot{\Omega}=[A, \Omega]+e^{\Omega} \dot{\Omega} e^{-\Omega} .
$$

then, we proceed to exchange $\Omega$ by $\lambda \Omega$ and integrate between zero to one, one has

$$
\int_{0}^{1} d \lambda e^{\lambda \Omega} \dot{\Omega} e^{-\lambda \Omega}=\dot{\Omega}+[\Omega, A]
$$


Finally, identifying the left side of the Eq. (3.16) as

$$
A=\int_{0}^{1} d \lambda e^{\lambda \Omega} \dot{\Omega} e^{-\lambda \Omega}
$$

then, the Eq. (3.13) can be written as

$$
\dot{\Omega}=A+[A, \Omega] .
$$

Now solve the Eq. (3.18) is a simple task. It can be shown by simple derivation that the final solution is

$$
\Omega(t)=\int_{0}^{t} d t_{1} A+\sum_{j=2}^{\infty} d t_{j} \cdots \int_{0}^{t_{2}} d t_{1}\left[A_{j},\left[\cdots\left[A_{2}, A_{1}\right] \cdots\right]\right]
$$

where $A_{j}=A\left(t_{j}\right)$. The first terms of the Eq. (3.19) are

$$
\Omega(t)=\int_{0}^{t} d t_{1} A+\int_{0}^{t} d t_{2} \int_{0}^{t_{2}} d t_{1}\left[A_{2}, A_{1}\right]+\int_{0}^{t} d t_{3} \int_{0}^{t_{3}} d t_{2} \int_{0}^{t_{2}} d t_{1}\left[A_{3},\left[A_{2}, A_{1}\right]\right]+\ldots
$$

Comparing Eq. (3.19) with Eq. (2.12) also shows that differ by the numerical coefficients, and that $\Omega(t)$ is no longer more expressed in terms of $\Omega(t)$. The operator $e^{\Omega}$ in Eq. (3.19) meets the criterion of Friederich [10], i. e., $\Omega(t)$ is an element of Lie.

The solution $Y(t)=e^{\Omega(t)}$ has many applications. For example, classical mechanics, quantum electrodynamics, quantum statistical mechanics,quantum field theory and group theory.

\section{EXAMPLE}

This section develops an application example of the solution of the movement of a particle in a curve, whose description is obtained from the Frenet-Serret equations of differential geometry. The Frenet-Serret equations [11] are given by the following differential equations

$$
\begin{gathered}
\frac{d \widehat{t}}{d s}=\kappa \widehat{n}, \\
\frac{d \widehat{n}}{d s}=\tau \widehat{b}-\kappa \widehat{t}, \\
\frac{d \widehat{b}}{d s}=-\tau \widehat{n},
\end{gathered}
$$

where, $\widehat{t}, \widehat{n}$ and $\widehat{b}$, are the tangent, normal, and binormal vectors in the curve, respectively; $\kappa$ and $\tau$ are the curvature and torsion of the curve and generally depend on the parameter of arc length $s$.

The set of Eq. (4.1) can be written as a matrix differential equation of the form:

$$
\frac{d Y(s)}{d t}=A(s) Y(s)
$$

where

$$
A(s)=\left[\begin{array}{ccc}
0 & \kappa & 0 \\
-\kappa & 0 & \tau \\
0 & -\tau & 0
\end{array}\right]
$$


and

$$
Y(s)=\left[\begin{array}{ccc}
t_{x} & t_{y} & t_{z} \\
n_{x} & n_{y} & n_{z} \\
b_{x} & b_{y} & b_{z}
\end{array}\right]
$$

Also, the matrix can be written in terms of the two matrix $M_{k}$ known as the infinitesimal generators of the three-dimensional rotations [12],

$$
A(s)=-\kappa M_{3}+\tau M_{1},
$$

where

$$
M_{1}=\left[\begin{array}{ccc}
0 & 0 & 0 \\
0 & 0 & 1 \\
0 & -1 & 0
\end{array}\right] ; M_{2}=\left[\begin{array}{ccc}
0 & 0 & 1 \\
0 & 0 & 0 \\
-1 & 0 & 0
\end{array}\right] ; M_{1}=\left[\begin{array}{ccc}
0 & -1 & 0 \\
1 & 0 & 0 \\
0 & 0 & 0
\end{array}\right],
$$

which satisfy the following commutation rules:

$$
\left[M_{i}, M_{j}\right]=\varepsilon_{i j k} M_{k} .
$$

These define the Lie algebra of the group SO3.

Luna and Chavoya solved Eq. (3.18) as genuine exponential in the case where the matrix $A(s)$ not dependent on the parameter $s$, and also exploiting the property of linear operators idempotent; as applied to a subgroup of the group single parameter SO3corresponding to rotations about an axis fixed direction.

Consider now that the matrix $A(s)$ itself depends on the parameter $s$ but that the torsion and curvature are equal; to exploit the property mentioned idempotent operators.

If $\kappa=\tau$, the Eq. (4.5) remains as

$$
A(s)=\kappa\left(M_{1}-M_{3}\right)
$$

The calculation of the matrix $\Omega(s)$ is as follows. Calculating the terms of Eq. (3.19) we have

$$
\int_{0}^{s} d s_{1} A_{1}=\left(M_{1}-M_{3}\right) \int_{0}^{s} \kappa\left(s_{1}\right) d s_{1}=\left(M_{1}-M_{3}\right) g(s)
$$

where $g(s)=\int_{0}^{s} \kappa\left(s_{1}\right) d s_{1}$. Then,

$$
\begin{gathered}
\int_{0}^{s} d s_{2} \int_{0}^{s_{2}} d s_{1}\left[A_{1}, A_{2}\right]=\int_{0}^{s} d s_{2} \int_{0}^{s_{2}} d s_{1}\left[\kappa_{1}\left(M_{1}-M_{3}\right), \kappa_{2}\left(M_{1}-M_{3}\right)\right] \\
=\int_{0}^{s} d s_{2} \int_{0}^{s_{2}} d s_{1}\left(\kappa_{2} \kappa_{1}\right)\left[\left(M_{1}-M_{3}\right)^{2}-\left(M_{1}-M_{3}\right)^{2}\right]=0
\end{gathered}
$$

therefore, the following terms in the series development of the Eq. (3.19) are also zero.

Now the matrix $\Omega(s)$ is left on the form

$$
\Omega(s)=g(s)\left(M_{1}-M_{3}\right)=\frac{A(s)}{\kappa} g(s)=g(s) \Omega_{1}(s),
$$


where

$$
\Omega_{1}(s)=\left[\begin{array}{ccc}
0 & 1 & 0 \\
-1 & 0 & 1 \\
0 & -1 & 0
\end{array}\right] .
$$

It can be shown directly, the powers of $\Omega(s)$ in terms of the powers of $g(s)$ fulfill the properties given below

$$
\begin{aligned}
& \text { a) } \Omega^{2 n+1}(s)=(-2)^{n} g^{2 n+1}(s) \Omega_{1} ; n=0,1,2, \ldots, \\
& \text { b) } \Omega^{2 n+2}(s)=(-2)^{n} g^{2 n+2}(s) \Omega_{2} ; n=0,1,2, \ldots, \\
& \text { c) } \Omega_{2}(s)=\Omega_{1}^{2}(s)=\left[\begin{array}{ccc}
-1 & 0 & 1 \\
0 & -2 & 0 \\
1 & 0 & -1
\end{array}\right], \\
& \text { d) }\left[\Omega_{1}, \Omega_{2}\right]=0 .
\end{aligned}
$$

$$
e^{\Omega(s)}=\sum_{n=0}^{\infty} \frac{\Omega^{n}(s)}{n !}=I+\Omega_{1} \sum_{n=0}^{\infty} \frac{(-1)^{n} g^{2 n+1}(s)}{(2 n+1) !} 2^{n}+\Omega_{2} \sum_{n=0}^{\infty} \frac{(-1)^{n} g^{2 n+2}(s)}{(2 n+2) !} 2^{n} .
$$

Identifying with sine and cosine series, finally we have

$$
Y(s)=e^{\Omega(s)}=I+\frac{1}{\sqrt{2}} \Omega_{1} \sin (\sqrt{2} g(s))+\frac{1}{2} \Omega_{2}(1-\cos (\sqrt{2} g(s))) .
$$

This expression that corresponds to the desired solution, and which when multiplied by $Y(0)$, provides the tangent, normal and binormal vectors for all value of the parameter $s$.

\section{CONCLUSIONS}

The present method has the virtue of allowing solve the differential equation easily, displaying a $\Omega(t)$ as a function of $A(t)$ and multiple commutators required at all levels in the series. This series can be cut after some few terms of it depending on the problem. If we also take advantage of the possibility of idempotent $\Omega(t)$, series expansion of the exponential of this operator converges and the solution of the differential equation remains a function of a matrix basis that leads to functions sine, cosine or sometimes hyperbolic functions of sines and cosines. It is considered that this method can potentially be particularly useful in many branches of the physics, and the general solution of the Cambell-Baker-Hausdorff's problem.

\section{REFERENCES}

[1] J. Schwinger, Phys. Rev. 74 (1948),1439-1461.

[2] F. J. Dyson, Phys. Rev. 75 (1949), 486-502.

[3] R. P. Feynman, J. Phys. Rev. 84 (1951),108-128.

[4] H. Luna-García, O. Chavoya-Aceves, Rev. Mex. Fis. 35(4) (1980),680-690.

[5] W. Magnus, Comm. Pure Appl. Math., Vol. VII, (1954),649-673.

[6] Abramowitz and Stegun, Handbook of Mathematical Functions, Dover Publications, USA (1964).

[7] R. M. Wilcox, J. Math. Phys. 8 (1967),962-982.

[8] B. Mielnik, J. Plebañski, Ann. Inst. Henri Poincaré, Vol. XII, No. 3, (1970), 215-254.

[9] E. B. Dynkin, Doclady Akad. Nauk, SSSR (N. S.) 57(1947), 323-326. 
[10] K. O. Friederich, Comm. Pure Appl. Math., Vol. VI, (1953),1-72.

[11] D. Danielson, Vectors and Tensors in Engineering and Physics, 2nd. Ed., Addison-Wesley,USA (1992).

[12] H. Goldstein. Classical Mechanics, 2nd. Ed., Addison-Wesley,USA (2000).

Área de Física Atómica Molecular Aplicada, CBi, UAM-A, Av. San Pablo No. 180, Col. Reynosa, Azcapotzalco, México, D. F. 02200, México.

E-mail address: 5553972854.hl@gmail.com 